Math. Proc. Camb. Phil. Soc. (1998), 123, 41

\title{
Arc index and the Kauffman polynomial
}

\author{
BY HUGH R. MORTON \\ Department of Mathematical Sciences, University of Liverpool, PO Box 147, \\ Liverpool L69 $3 B X$ \\ AND ELISABETTA BELTRAMI* \\ Dipartimento di Matematica, Università di Pisa, Via F. Buonarroti 2, 56127 Pisa, \\ Italy
}

(Received 28 May 1996)

Abstract

The arc index $\alpha(L)$ of a link $L$ is shown by a direct combinatorial argument to be related to $\operatorname{spr}_{v}\left(F_{L}(v, z)\right)$, the Laurent degree of its Kauffman polynomial, by the inequality

$$
\alpha(L) \geqslant \operatorname{spr}_{v}\left(F_{L}(v, z)\right)+2
$$

Equality is conjectured for alternating links.

\section{Introduction}

The arc index $\alpha(L)$ of a link $L$ is the smallest number of arcs needed to draw $L$ when each arc lies with its ends on the axis in a coordinate half-plane for cylindrical polar coordinates. Such presentations were originally described by Brunn[2], but have recently been revived by work of Birman and Menasco in connection with finding braid presentations for reverse-string satellites of $L$, such as the reverse parallels and doubles [1], and have been studied further by Cromwell and Nutt[3], [5], [11].

In this paper we find a bound for $\alpha(L)$ in terms of the Laurent degree (spread) of the Kauffman polynomial $F_{L}(v, z)$ in the variable $v$ which is related to the framing change for the framed version of $F$. The explicit bound is

$$
\alpha(L) \geqslant \operatorname{spr}_{v}\left(F_{L}(v, z)\right)+2 .
$$

We conjecture that equality holds for alternating links, where it is known [14] that $\operatorname{spr}_{v}\left(F_{L}\right)=c(L)$, the crossing number of $L$. This conjecture would follow from the conjecture of Nutt that $\alpha(L) \leqslant c(L)+2$.

The result bears some resemblance to the Morton-Franks-Williams bound for braid index in terms of the analogous variable in the Homfly polynomial, [6], [9]. Indeed, Nutt[11], [12] has already used results of Rudolph[13] connecting the Kauffman polynomial of $L$ and the Homfly polynomial of the reverse parallels of $L$, combined with the relation between the arc index of $L$ and the braid index of reverse parallels, and the MFW bound to give the weaker bound $\alpha(L) \geqslant \operatorname{spr}_{v}\left(G_{L}(v, z)\right)+2$, where $G_{L}$ is $F_{L}$ with coefficients reduced $\bmod 2$, in the case where $L$ is a knot.

Here we present a simple direct combinatorial proof of our result, which involves the Kauffman polynomial itself, rather than its $\bmod 2$ reduction, and holds for all

\footnotetext{
* Dottorato di ricerca.
} 
links. We first prove a more general theorem about the framed Kauffman polynomial for links constructed from a special kind of tangle, in which simple arcs are stacked inside a disc and connected outside the disc by arcs without further crossings. Such tangles arise from the arc-presentation of a link, and are also used [10] as basis elements for the Birman-Wenzl algebra.

As a corollary, the inequality proved in [4] for alternating knots $\alpha(K) \geqslant c(K)+2$, where $c(K)$ is the crossing number of $K$, extends to alternating links; the conjecture made in [5], that $\alpha(L)=c(L)+2$ for all alternating links, is still open.

\section{The Kauffman polynomial}

We shall consider the Dubrovnik framed version of the Kauffman polynomial. The Dubrovnik polynomial $\mathscr{D}$ is a function defined on link diagrams with values in the ring of Laurent polynomials $\mathbf{Z}\left[v^{ \pm 1}, z^{ \pm 1}\right]$. It is characterized by the following basic properties $[\mathbf{7}, \mathbf{8}]$ :

$(\mathbf{S R})$ skein relation: $\mathscr{D}_{K_{+}}-\mathscr{D}_{K_{-}}=z \mathscr{D}_{K_{0}}-z \mathscr{D}_{K_{\infty}}$, where the diagrams $K_{+}, K_{-}, K_{0}, K_{\infty}$ differ only as shown.

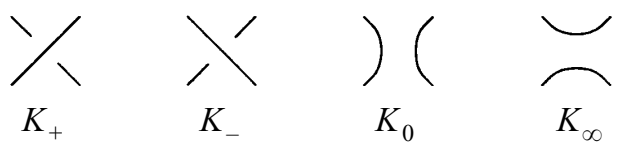

(C) behaviour with curls: $\mathscr{D}_{K_{n}}=v \mathscr{D}_{K}$ and $\mathscr{D}_{K_{p}}=v^{-1} \mathscr{D}_{K}$, where $K_{n}$ and $K_{p}$ are given from $K$ by adding a negative or positive curl as shown.

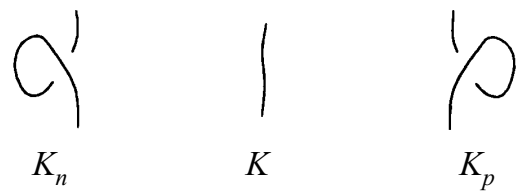

(N) normalization: $\mathscr{D}_{O}=1$, where $O$ is the diagram of the unknot without any crossings.

The Dubrovnik polynomial is a regular isotopy diagram invariant, and thus a framed link invariant.

Remark 1. If $K=K^{\prime} \amalg O$ is a split link diagram and the component $O$ is the unknot with no self-crossings then $\mathscr{D}_{K}=\delta \mathscr{D}_{K^{\prime}}$, where $\delta=1+\left(v^{-1}-v\right) z^{-1}$.

Remark 2. If $U_{l}$ is a diagram of the unlink with $l$ components having no crossings then $\mathscr{D}_{U_{l}}=\delta^{l-1}$.

Let $K$ be a diagram of an oriented link $L$. The number of crossings in $K$ counted with their $\operatorname{sign} \epsilon$, as shown, is called the writhe of $K$, and is denoted by $w(K)$.

$$
\% \epsilon=+1, \lambda \epsilon=-1
$$

Define the Laurent polynomial $D_{L}$ by

$$
D_{L}(v, z)=v^{w(K)} \mathscr{D}_{K}(v, z) .
$$

Theorem 3 (Lickorish $[8]$ ). The polynomial $D_{L}$ is an oriented link invariant, related to the Kauffman polynomial $F_{L}$ by the equation $D_{L}(v, z)=(-1)^{|L|-1} F_{L}\left(-i v^{-1}, i z\right)$, where $|L|$ denotes the number of components of $L$. 
In particular, notice that the Laurent degree in the variable $v$, that is the difference between the highest and lowest powers of $v$, is the same for each of $\mathscr{D}, D$ and $F$. This allows us to deal with the framed version of Dubrovnik in place of the Kauffman polynomial.

\section{Stacked tangles}

We now consider a particular kind of tangle. Take a simple closed curve and mark $2 d$ points on it. We refer to this marked curve as the frame of the tangle. Imagine $d$ discs stacked up, each with the frame as boundary, and each containing a simple arc which joins two of the marked points on the frame. We shall call the resulting tangle $S_{d}$, viewed from above, a stacked tangle. Such a tangle has $d$ simple arcs inside the frame, each lying at a different level, as in the example below.

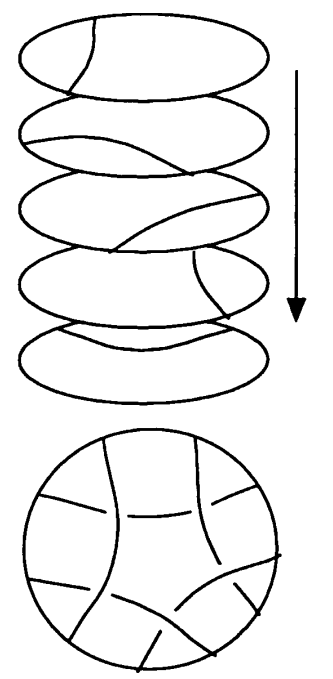

A stacked tangle with 5 arcs.

A stacked tangle has no closed components, the individual arcs are unknotted and can be totally ordered by the depth of the leaves in which they lie. We will consider such tangles up to regular isotopy. They are then determined by the pairs of endpoints joined by the are in each level. If the endpoints of two arcs interleave on the frame then we can draw the tangle so that these arcs cross in only one point; if their endpoints do not interleave then we can arrange that the arcs do not cross at all.

Given a tangle $T$ with $d$ ares, we will refer to a link diagram $K$ as a simple closure of $T$ when it consists of $T$ and $d$ arcs outside the frame having no further crossings. Here is a picture of a simple closure of the stacked tangle shown earlier.

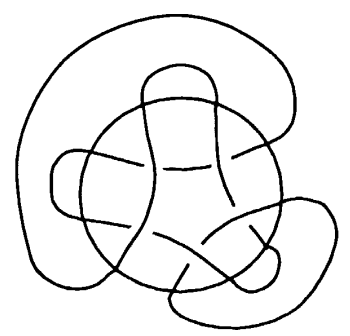


We say that one of the ares lying outside the frame is a cap if it joins two consecutive marked points on the frame. A simple closure always has at least one cap.

Theorem 4. Let $K$ be a simple closure of a stacked tangle $S_{d}$ with d arcs. Write its framed Dubrovnik polynomial as $\mathscr{D}_{K}(v, z)=\sum_{i=m}^{M} a_{i}(z) v^{i}$, with $a_{M} \neq 0, a_{m} \neq 0$. Then $M \leqslant d-1, m \geqslant 1-d$.

Proof. By induction on $(d, n)$, where $n$ is the number of crossings in $K$.

Base of the Induction. If $d=1$ then $K$ is the unknot without crossings, so $M=$ $m=0$.

Inductive Step. Assume the theorem is true for $\left(d^{\prime}, n^{\prime}\right)<(d, n)$ and let $K$ be a simple closure of a stacked tangle $S_{d}$ with $n$ crossings. Let $f$ be a cap outside $S_{d}$, joining points $P, Q$ of the frame. Consider the ares in $S_{d}$ with $P$ and $Q$ as end points. There are two possible cases.

(i) A single $\operatorname{arc} g$ joins $P$ and $Q$. Then $K=K^{\prime} \amalg O$, where $O$ is made from $f$ and $g$, and $K^{\prime}$ is a simple closure of a stacked tangle with $d-1$ arcs. The result will follow by the induction hypothesis and Remark 1.

(ii) There are two arcs, $a$ joining $P$ to $P^{\prime}$ and $b$ joining $Q$ to $Q^{\prime}$. We may suppose that $a$ lies at a higher level than $b$, which we will write as $a>b$. In this case, either (I) $a$ and $b$ are at adjacent levels, or (II) there are other ares between them, $a>h_{1}>\ldots>h_{r}>b$.

(I) Replace the part of the diagram consisting of $a, f$ and $b$, which will look like one of the three pictures here,
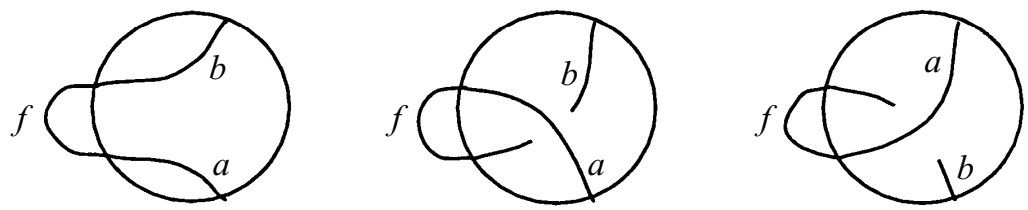

by a simple arc inside the frame joining $P^{\prime}$ to $Q^{\prime}$ at the same level as $a$, as indicated.

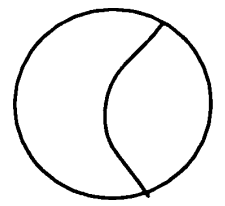

The new diagram $K^{\prime}$ is now a simple closure of a stacked tangle with $d-1$ arcs, so the inductive hypothesis applies to give $\mathscr{D}_{K^{\prime}}(v, z)=\sum_{i=m^{\prime}}^{M^{\prime}} a_{i}(z) v^{i}$, with $M^{\prime} \leqslant d-2, m^{\prime} \geqslant$ $2-d$. If the arcs $a$ and $b$ do not cross then $K^{\prime}$ is regularly isotopic to $K$ and so $\mathscr{D}_{K^{\prime}}=$ $\mathscr{D}_{K}$. Otherwise $a, f$ and $b$ form a curl, and so $\mathscr{D}_{K}=v^{ \pm 1} \mathscr{D}_{K^{\prime}}$ by property (C). In either case the result will follow.

(II) Use a subsidiary induction on the difference in level between the ares $a$ and $b$ joined by a cap. The base of the induction is established by (I). For the induction step consider the arc $h_{r} \neq a$ in the level immediately above $b$. If $b$ and $h_{r}$ do not interleave then we may interchange their levels without altering $K$, and the result follows from the subsidiary induction, since $a$ and $b$ are now closer. 
Otherwise, write $K=K_{+}$and write $K_{-}$for the diagram in which the levels of $b$ and $h_{r}$ are interchanged. Because the arcs lie in adjacent levels in $K$ these two diagrams simply differ in the neighbourhood of one crossing, as for the skein relation at this crossing. The diagrams $K_{0}$ and $K_{\infty}$ also arise as simple closures of stacked tangles with $d$ arcs, differing from $K$ by the choice of two arcs in adjacent levels in place of $b$ and $h_{r}$ as shown.

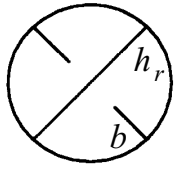

$K_{+}=K$

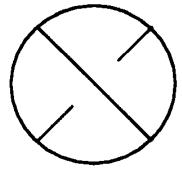

K

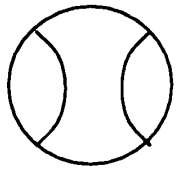

$K_{0}$

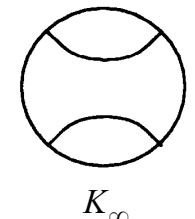

$K_{\infty}$

In $K_{-}$the arcs joined by the cap $f$ are closer than in $K$, while $K_{0}$ and $K_{\infty}$ have fewer crossings than $K$, so the inequalities hold for each of these by the induction hypotheses. Since $\mathscr{D}_{K_{+}}=\mathscr{D}_{K_{-}}+z\left(\mathscr{D}_{K_{0}}-\mathscr{D}_{K_{\infty}}\right)$ the inequality for $\mathscr{D}_{K}$ follows.

\section{Arc-presentation and arc index}

We consider an open book decomposition of $S^{3}$ which has dises as pages and an unknotted circle as binding. An arc-presentation of a $\operatorname{link} L$ is an embedding of $L$ in a set of finitely many of these discs so that it meets each of them in a single simple arc whose endpoints are on the binding circle. In the illustration given here the binding circle is drawn as the axis for cylindrical polar coordinates.

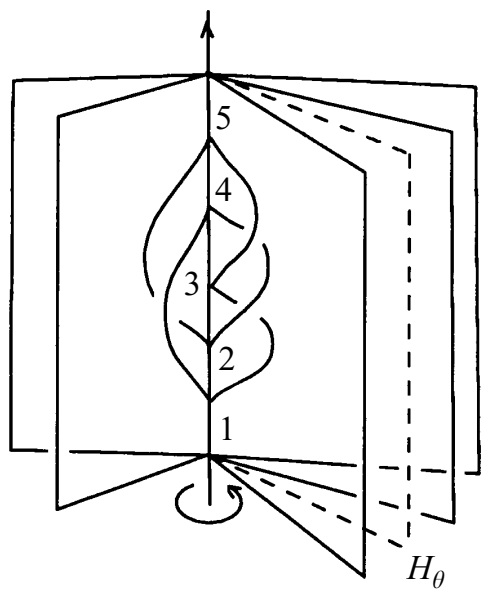

The minimum number of pages, or equivalently the minimum number of intersections between $L$ and the binding, required to present $L$ in this way is a link invariant called the arc index of $L$, and is denoted by $\alpha(L)$.

We can convert an embedding like that shown into a planar diagram, illustrated below, by proceeding as follows. Orient the binding and label the pages using polar coordinates in order to give $S^{3}$ the right-hand orientation. Choose a page $H_{\theta}$ at angle $\theta$, which does not contain arcs of $L$. Draw the binding as a simple loop $\gamma$ on the plane, orient it clockwise and mark the points of its intersection with $L$ on $\gamma$. Draw the ares inside $\gamma$, starting with the one next to $H_{\theta}$ and proceed consecutively around the binding, passing successive arcs under previous ones, if necessary. The diagram that 
appears is what you can see on the plane by closing the open book in which $L$ is embedded with $H_{\theta}$ as cover.

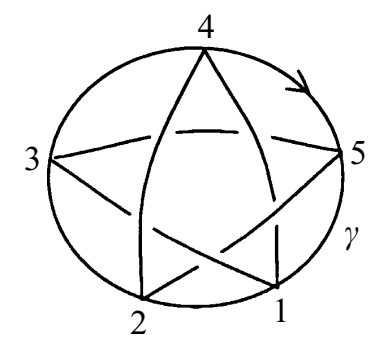

Now modify this diagram slightly to obtain a simple closure of a stacked tangle. Take a simple closed curve $\phi$ inside $\gamma$, but near enough to it so that no crossings lie between them. The circle $\phi$ is the frame of a stacked tangle $S_{d}$, where $d$ is the number of arcs in the starting arc-presentation of $L$.

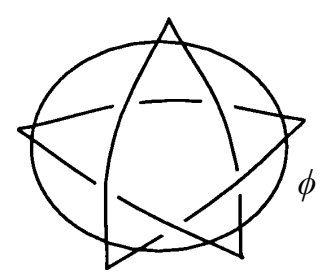

The result is a diagram $K$ of $L$ which is a simple closure of a stacked tangle with $d$ arcs, where the arcs outside the frame consist of $d$ caps. Write $\mathscr{D}_{K}(v, z)=\sum_{i=m}^{M} a_{i}(z) v^{i}$. Theorem 4 then shows that $M \leqslant d-1$ and $m \geqslant 1-d$ so that $\operatorname{spr}_{v}\left(\mathscr{D}_{L}\right)=M-m \leqslant$ $2 d-2$. By taking a minimal arc presentation we get $\operatorname{spr}_{v}\left(\mathscr{D}_{L}\right) \leqslant 2 \alpha(L)-2$. We can improve this bound considerably by further application of Theorem 4 .

Theorem 5. For every link $L$ we have $\operatorname{spr}_{v}\left(F_{L}\right) \leqslant \alpha(L)-2$.

Proof. Consider an arc-presentation of $L$ on $d$ arcs, leading to a diagram $K$ which is a simple closure of a stacked tangle on $d$ arcs, as described above. The curves in $K$ outside the frame consist of $d$ caps.

Let $f$ be any of these caps and let $a$ and $b$ be the arcs inside $\phi$ that meet $f$, with $a>b$. Alter the arcs $a$ and $b$ without changing their levels, so that they each meet the other end of the cap $f$. This will introduce an overcrossing of $a$ over $b$, giving a new diagram $K^{\prime}$, which is again a simple closure of a stacked tangle with $d$ arcs.

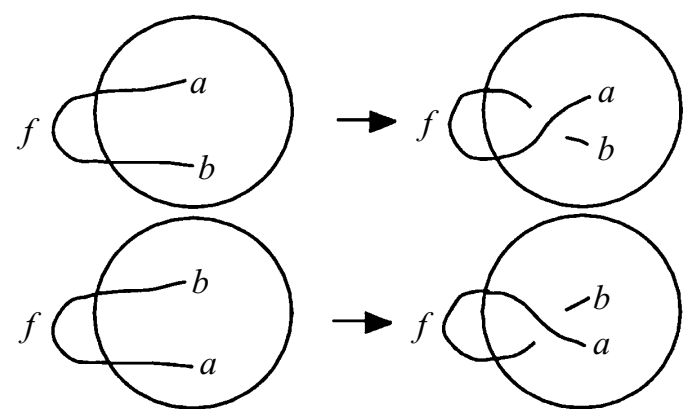

Moreover $K^{\prime}$ is still a diagram of $L$ and differs from $K$ only by a curl, positive or negative. Each of the $d$ caps in $K$ will permit a similar modification. Suppose that $p$ 
of the caps yield a positive curl, and the remaining $d-p$ yield a negative curl. Write $K_{\text {pos }}$ for the diagram obtained by inserting the $p$ positive curls in $K$ as above, and $K_{n e g}$ similarly by inserting the $d-p$ negative curls. Then $\mathscr{D}_{K_{p o s}}=v^{-p} \mathscr{D}_{K}$ and $\mathscr{D}_{K_{\text {neg }}}=$ $v^{d-p \mathscr{D}_{K}}$.

Now the diagrams $K_{\text {pos }}$ and $K_{\text {neg }}$ are still simple closures of stacked tangles with $d$ ares. Theorem 4 applied to $K_{\text {neg }}$ then gives $M+d-p \leqslant d-1$ and applied to $K_{\text {pos }}$ gives $m-p \geqslant 1-d$. Subtract these, to get $M-m \leqslant d-2$. Choose a presentation with

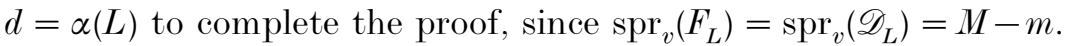

Using Theorem 5 and an observation [4] from Thistlethwaite's work on alternating links, we can deduce the following:

Corollary 6. Let $L$ be an alternating link with crossing number $c(L)$; then $\alpha(L) \geqslant$ $c(L)+2$.

Remark 7. Consider the minimal arc-presentation of the right trefoil $T$ on $\alpha(T)=$ 5 ares shown above. It produces a diagram $K$ with writhe, or equivalently blackboard framing, $w(K)=+3$. The framed Dubrovnik polynomial of $K$ has $M=2, m=-1$. By adding curls to $K$ we can change its framing, but only a small number of framed diagrams of the knot $T$ can be realized as a simple closure of a stacked tangle with this minimum number of ares. For example the polynomial $\mathscr{D}$ for the diagram of $T$ with framing 0 has $m=2$ and $M=5>\alpha(T)-1=4$, and hence we need at least 6 ares, by Theorem 4, (this number of ares is actually enough, as seen in the picture below).

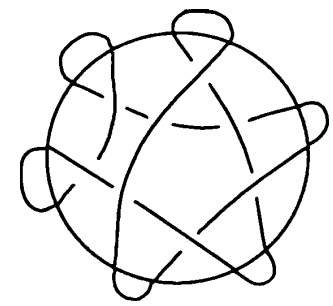

Similar restrictions arise for any link $L$. Write $s_{v}(L)$ for $\operatorname{spr}_{v}\left(F_{L}\right)=\operatorname{spr}_{v}\left(\mathscr{D}_{L}\right)$ and suppose that $L$ can be presented as the simple closure $K$ of a stacked tangle with $d$ arcs, closed with $d$ caps. This diagram has framing determined (for a knot at least) by the writhe $w(K)$ and will arise from some arc-presentation on $d$ arcs. If we present $L$ by some other stacked tangle $K^{\prime}$ again with $d$ arcs we can alter the framing, but over a restricted range only. Write $W_{d}(L)$ and $w_{d}(L)$ for the maximum and minimum values which can be obtained for presentations using $d$ arcs. Then the range $W_{d}(L)-w_{d}(L)$ satisfies

$$
d \leqslant W_{d}(L)-w_{d}(L) \leqslant 2 d-2-s_{v}(L) .
$$

The first part of the inequality comes from the difference of $d$ in framing between the diagrams $K_{\text {pos }}$ and $K_{\text {neg }}$ constructed above by inserting suitable curls near the caps in $K$.

The second part comes from the control on $W_{d}(L)$ given by Theorem 4 , namely that the highest degree in $\mathscr{D}_{K^{\prime}}$ cannot exceed $d-1$. In terms of one known polynomial $\mathscr{D}_{K}$ with highest degree $M_{K}$ (and lowest degree $m_{K}=M_{K}-s_{v}(L)$ ) we have $M_{K}+\left(W_{d}(L)-w(K)\right) \leqslant d-1$. This gives an upper bound for $W_{d}(L)$, which is complemented by the bound $m_{K}-\left(w(K)-w_{d}(K)\right) \geqslant 1-d$. Subtract these, to get the second part of the inequality. 
Given a diagram $K$ for $L$ which is a simple closure of a stacked tangle on $d$ ares with writhe $w(K)$ it is easy to add an extra arc and cap to get presentations of $L$ on $d+1$ arcs, with any of the choices $w(K), w(K) \pm 1$ for writhe. It follows from the bounds just established that $W_{d}(L)=C+d$ and $w_{d}(L)=c-d$ when $d \geqslant d_{0} \geqslant \alpha(L)$, for some constants $C, c$ and $d_{0}$ depending on $L$. This may be compared with the form of the restrictions established by Nutt for the braid index of a reverse parallel for a knot in terms of its framing, using the Homfly polynomial, [11]. His result requires a nontriviality assumption for some Homfly polynomial, while our result holds for all knots. It may thus be possible to strengthen his result by a more precise inspection of the nature of the arc presentation of $L$ derived from a braid presentation of a given reverse parallel.

\section{REFERENCES}

[1] J. S. Birman and W. W. Menasco. Special positions for essential tori in link complements. Topology 33 (1994), 525-556.

[2] H. Brunn. Uber verknotete Kurven. Mathematiker-Kongresses Zurich 1897 (Leipzig, 1898), $256-259$.

[3] P. R. Cromwell. Embedding knots and links in an open book I: basic properties. Topology and its Applications 64 (1995), 37-58.

[4] P. R. Cromwell. Are presentations of knots and links. Proc. 1995 Warsaw knot theory conference, to appear.

[5] P. R. Cromwell and I. J. Nutt. Embedding knots and links in an open book II : bounds on arc index. Math. Proc. Camb. Phil. Soc. 119 (1996), 309-319.

[6] J. Franks and R. F. Williams. Braids and the Jones-Conway polynomial. Trans. Amer. Math. Soc. 303 (1987), 97-108.

[7] L. H. Kauffman. An invariant of regular isotopy. Trans. Amer. Math. Soc. 318 (1990), $417-471$.

[8] W. B. R. Lickorish. Polynomials for links. Bull. London Math. Soc. 20 (1988), 558-588.

[9] H. R. Morton. Seifert circles and knot polynomials. Math. Proc. Camb. Phil. Soc. 99 (1986), 107-109.

[10] H. R. Morton and P. Traczyk. Knots and algebras. In 'Contribuciones Matematicas en homenaje al profesor D. Antonio Plans Sanz de Bremond', ed. E. Martin-Peinador and A. Rodez Usan, University of Zaragoza, (1990), 201-220.

[11] I. J. Nutr. Braid index of satellite links, Ph.D. thesis (University of Liverpool, 1995).

[12] I. J. Nutr. Arc index and the Kauffman polynomial. J. Knot Theory Ramif. 6 (1997), 61-77.

[13] L. Rudolph. A congruence between link polynomials. Math. Proc. Camb. Phil. Soc. 107 (1990), 319-327.

[14] M. B. Thistlethwaite. Kauffman polynomial and alternating links. Topology 27 (1987), $297-309$. 